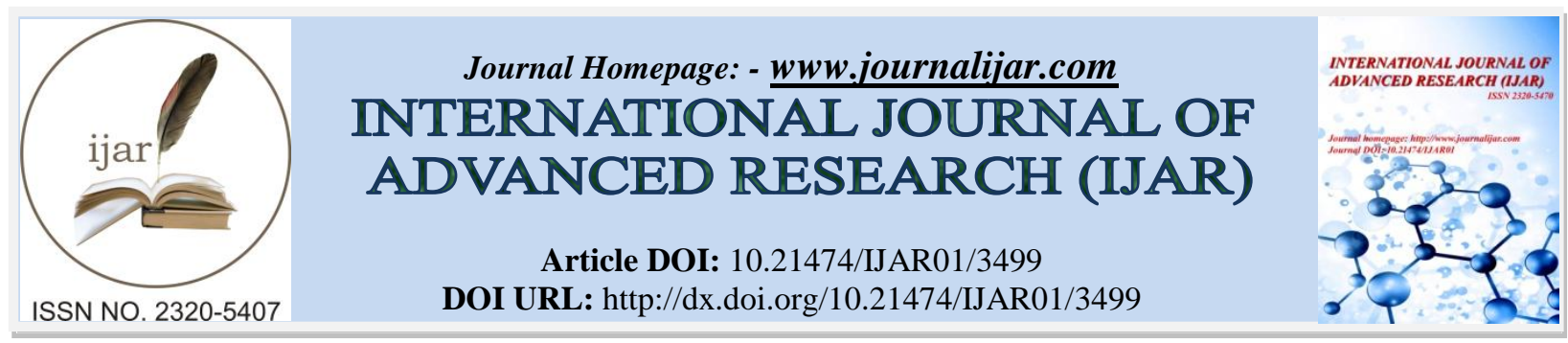

RESEARCH ARTICLE

\title{
LA GOUVERNANCE DES DESTINATIONS TOURISTIQUES DANS LA NOUVELLE POLITIQUE TOURISTIQUE MAROCAINE: ENTRE CONSTRUCTION INSTITUTIONNELLE ET REALITE OPERATIONNELLE.
}

Achaba Allal et Bendou Abdelaziz.

Enseignants Chercheurs à l'Ecole Nationale de Commerce et de Gestion Agadir, Université Ibn Zohr (Maroc)

\section{Manuscript Info}

Manuscript History

Received: 06 January 2017

Final Accepted: 04 February 2017

Published: March 2017

Key words:-

Tourism Destination, Tourism Policy, Strategic Management, Governance,

Tourism Destination Governance, Vision 2020.

\begin{abstract}
Promoted since the early sixties as a priority sector in the strategy of socio - economic development, the Moroccan tourism sector, in view of the achievements and the great potential of tourism of the country and of the important growth of world tourism Will undergo a radical change after the Year 2000 in its development policy by moving from a tourism policy mainly promotional by state to the era of strategy in the framework of ambitious, voluntarist and integrated development visions based on contractualization and public-private partnership. These new visions placing tourism as a driving force in the development of the country have focused on strengthened governance to ensure the steering of the strategy and ensure the effective implementation of strategies and action plans.

The purpose of this article is to present an analytical reading of the new model of tourism governance in Morocco in order to characterize it and to analyze its effectiveness and the main limitations to its operational implementation. On the methodological level, we have mobilized the theories and models of strategic management and the governance of tourist destinations and a documentary analysis backed up by a qualitative study by semi-directive interviews with the main actors of the sector.
\end{abstract}

Copy Right, IJAR, 2017,. All rights reserved.

\section{Introduction:-}

Depuis que le tourisme s'est confirmé comme un secteur très important pour le développement socio-économique des pays et au vue des perspectives prometteuses de son développement futur les questions du management, de la compétitivité des destinations touristiques a retenue l'attention tant des chercheurs, des professionnels, des gouvernements que des organismes internationaux. L'environnement turbulent (Graulier2000) et concurrentiel (Chazaud, 1999) mais aussi indifférencié (Rauche, 2000) dans le quel s'inscrivent les destinations touristiques ont remis en cause les politiques touristiques classiques basées sur des logiques de promotion et de commercialisation tout azimut et ont imposé aux acteurs et destinations touristiques de réfléchir et de mettre en place de nouvelles stratégies tenant compte de leurs attributs attractifs et des lourdes mutations qui s'opèrent sur le marché. De même, les destinations touristiques étant un complexe d'acteurs autonomes et indépendants du point de vue organisationnel mais en principe fonctionnellement interdépendants dans la chaine de valeur de production touristique, leur management repose sur une nécessaire mobilisation et coordination d'acteurs à logiques et intérêts divergents. Cette particularité conduit ce management à s'étendre pour s'insérer dans un contexte de gouvernance de la destination (Zhang et Zhu, 2014). Le concept de gouvernance des destinations touristiques malgré sa jeunesse a donné lieu à 
une importante littérature visant à explorer son champ, ses logiques et dimensions (Gerbaux, Boudières et Marc elpoil, 2004; Gerbaux et George-Marcelpoil, 2004; Gerbaux et Moreau, 1996; Gerbaux et Paillet, 1999, François et Marcelpoil, 2006; Marcelpoil, Marcelpoil et Langlois, 2006 ; Bensahel et Vles, 2007). De même, qu'il a donné lieu à des modèles théoriques cherchant à proposer des schémas d'organisation et du management de la gouvernance des destinations touristiques.

$\mathrm{Au}$ Maroc le tourisme représenté un secteur fondamental dans la dynamique de développement socioéconomique du pays. Depuis l'année 2000 ce secteur va être érigé en locomotive de développement et une ambitieuse stratégie de développement va lui être consacrée rompant avec les politiques basées sur la seule promotion étatique engagées depuis le milieu des années 60. Cette nouvelle stratégie baptisée l'ère des visions (Vision 2010 et Vision 2020) va miser sur la gouvernance comme pilier du pilotage stratégique et de la mise en œuvre des stratégies et des plans d'action territoriaux qui en découlent.

Notre propos dans cet article et de mener unerecherche exploratoire pour tenter d'une part, d'analyser et de caractériser le nouveau modèle de gouvernance des destinations touristiques au Maroc et d'autre part, de jauger de son opérationnalisation et de ses limites à quatre années de l'horizon stratégique retenu. Pour ce faire nous avons d'une part mobilisé les principales théories et les principaux modèles du management stratégique et de gouvernance des destinations touristiques et sur le plan empirique nous avons allié pour la collecte des données l'exploitation de nombreux documents officiels de ce nouveau modèle de gouvernance et les données publiées sur sa mise en œuvre (statistiques, CPN, CPR, PDRT, études, articles de presse et publications dans les sites web des organisme touristiques nationaux et régionaux et bibliographie), une étude qualitative par entretiens semi-directifs, en face à face, auprès d'acteurs du secteur, les rapports des ateliers que nous avons animés au profit de l'AIH et enfin la participation active à diverses réunions, séminaires et manifestations publiques ou privées organisées par les acteurs au niveau national et régional.

\section{La politique touristique au Maroc : des programmes aux Visions:- Evolution de la politique touristique au Maroc:-}

L'analyse du système de management et de gouvernance du tourisme dans la destination Maroc nous oblige à revenir sur l'évolution de la politique touristique dans le pays et sa caractérisation. La Politique touristique est une expression générique qui donne lieu à diverses interprétations. Avec l'évolution du tourisme cette expression a connu aussi une nette évolution dans son contenu. Bécherel (2001) distingue quatre étapes différentes dans la formulation de la politique touristique. Au cours de la première étape, la politique touristique était orientée vers la «promotion» à travers la mise en place d'attractions publicitaires par les autorités touristiques. Avec l'affirmation du secteur touristique comme secteur économique significatif et porteur, la politique touristique s'est orientée plus vers l'optimisation du potentiel de production en multipliant les investissements dans l'offre d'hébergement et dans les infrastructures de bases et les actions de marketing et de promotion. Ces actions étaient généralement portées par les autorités nationales, régionales et locales faute d'un secteur privé fort ou à cause de son désintéressement. Dans cette deuxième étape, la politique touristique s'est orientée ainsi plus vers «la promotion et le produit». L'intensification de la concurrence sur le marché touristique mondial a amené les concepteurs de la politique touristique se concentrer sur la recherche de l'amélioration de la compétitivité du secteur en créant un cadre statutaire destiné à gérer, contrôler et améliorer la qualité et l'efficacité du secteur et à protéger les ressources. Actuellement, dans un marché touristique hyperconcurrentiel (Chazaud, 1999) et très évolutif tant de point de vue de l'offre que de la demande, la seule politique touristique basée sur la paire «promotion et produit» n'est plus suffisante pour assurer la compétitivité touristique. La stratégie et la planification stratégique deviennent des éléments clés de la politique touristique. Dans cette quatrième étape, les destinations doivent réfléchir en termes de stratégie, la politique doit être conçue de manière à attirer les marchés identifiés, à créer de la valeur à travers l'innovation, à encourager les partenariats entre les acteurs et à coordonner les actions et les initiatives. En outre, de nos jours, les parties intéressées et impliquées dans la gestion du secteur sont nombreuses et le sujet est bien plus vaste. «Compétitivité», «Viabilité» et «Durabilité» sont devenues les mots-clés de la vision moderne de développement du tourisme. Dans ce sens, La politique touristique a un double objectif : apporter un maximum de bénéfices aux intéressés d'une ville, d'une région ou d'un pays, tout en minimisant les impacts négatifs. Dans ce registre, Goeldner, Ritchie et McIntosh (2000) nous proposent une définition satisfaisante de l'expression «politique touristique»: «un ensemble de réglementations, règles, directives, objectifs de développement/de promotion et stratégies permettant de définir le cadre dans lequel sont prises les décisions collectives et individuelles qui affectent directement le développement du tourisme et les activités quotidiennes pour une destination donnée ». Ainsi, pour ces auteurs, le rôle le plus important de la politique touristique est de s'assurer qu'une destination a une idée claire de ce vers quoi elle se dirige ou de ce 
qu'elle cherche à devenir à long terme. En parallèle, elle doit avoir l'ambition de créer un climat favorable à la collaboration entre les nombreux acteurs du secteur touristique. Cela sous entend qu'une politique touristique efficace se doit de viser à offrir aux visiteurs des expériences de qualité, profitables aux parties intéressées de la destination tout en évitant de compromettre son intégrité sociale, culturelle et environnementale. Bécherel, sur la base de ces nouveaux enjeux a déterminé cinq domaines propres à la politique touristique de ce «nouvel âge» du tourisme : La politique de communication, la politique de produit, la politique environnementale, la compétitivité : qualité et efficacité, la stratégie: positionnement et ciblage.

La politique touristique marocaine n'a pas échappé aux mêmes règles d'évolution développées ci-dessus. En effet, l'activité touristique au Maroc a débuté certes sous le protectorat français mais c'est après l'indépendance du royaume que le secteur, sous l'impulsion des organismes internationaux (BM, ONU), va être retenu comme priorité de développement socioéconomique du pays. La politique touristique marocaine va connaitre deux principales phases dans son développement : la phase dites des programmes, allant de 1965 à la fin des années 90 caractérisée par au début un engagement total de l'Etat assurant le leadership du secteur et ensuite par son désengagement au profit du secteur privé tout en maintenant le rôle d'orientation. Et la phase dite des «visions» à partir de l'année 2001. En effet, l'attrait touristique du Maroc et ses caractéristiques géographiques, historiques et culturelles ainsi que le succès du tourisme international dans certains pays du pourtour de la Méditerranée vont être à la base de l'importance accordée au tourisme dans la stratégie de développement du pays au milieu des années soixante. C'est ainsi que le secteur va être érigé en priorité national dans les plans de développement successifs allant de 1965 à La fin des années 90. Cette période a été marquée par une politique touristique active, où le secteur public jouait un rôle dynamique en investissant massivement dans le secteur et en le réglementant. L'effort d'investissement et d'aménagement va se concentrer en premier lieu sur des zones prioritaires choisies en fonction de leur forte attraction touristique (Tanger, Al Hoceima, Restinga, Smir et Agadir) pour lesquelles des sociétés d'aménagement spécialisées vont être créées, puis sur les villes impériales disposant d'un patrimoine culturel ancestral (Marrakech, Rabat, Fès et Mekhnès). Cet effort va s'accompagner aussi par une action massive en matière de promotion. Les pouvoirs publics vont aussi par le biais d'organismes publics et semi-publics, tels que l'Office National Marocain du Tourisme (ONMT) et la Caisse de dépôt et de gestion (CDG), procéder à la création des premières unités d'hébergement dans les régions sous-équipées ou déshéritées. Un effort considérable a été aussi accordé à la variable ressource humaine avec la création des établissements de formation professionnelle dédiés au secteur. Les années 70 ont encore marqué cette orientation en instituant un code d'investissement spécifique au secteur dans lequel l'Etat a mis en place un dispositif de mesure incitant le privé à investir dans le secteur (prise en charge et garanti sous forme d'exonérations fiscales et de facilités de financement par le Crédit Immobilier et Hôtelier $(\mathrm{CIH})$ jusqu'à près de 75 $\%$ du coût des investissements des opérations à caractère touristique). La décennie 80 va connaitre, dans un contexte d'application du programme d'ajustement structurel, une orientation vers le développement des équipements hôteliers de catégorie moyenne de façon à répondre à la diffusion du tourisme de masse et une recherche de la diversification de l'offre et des produits par l'intégration de certains type de tourisme jusqu'alors marginalisés, tels que le tourisme familial, le tourisme de montagne et de sports d'hiver, le tourisme rural, le tourisme de nature, etc...

Le début des années 90 a été marqué par une crise d'essoufflement du tourisme (crise du golf, attentas terroriste de Marrakech). Dans ce contexte, une importante étude portant sur l'analyse du secteur et la mise en place d'une stratégie nationale d'aménagement touristique a été réalisée par le ministère. Cette étude, constatant le caractère vétuste du produit Maroc et la faiblesse de la qualité de service dans la grande majorité des établissements d'hébergement et son incapacité à rivaliser avec les nouvelles destinations touristiques va tracer les grandes lignes d'une stratégie de développement du tourisme au Maroc pour les années 1995-2010 et suggérer un programme d'action qui devraient l'accompagner. La mise en application des premières mesures du plan d'action de l'étude de la stratégie nationale d'aménagement touristique va rapidement donner ses fruits et l'année 2000 s'est soldé par des résultats forts encourageants (près de 4300000 non résidents dont 2275000 touristes étrangers de séjour, sans compter les croisiéristes, 22,3 millions de nuitées dont $50 \%$ dans les hôtels classés). En 2001, la Fédération du tourisme, membre de la Confédération générale des entreprises du Maroc (CGEM), et le gouvernement adoptent une stratégie de développement touristique sous la forme d'un contrat-programme 20012010, «le tourisme : une vision, un défi, une volonté », qui actualise et développe la stratégie de développement 1995-2010. Cette nouvelle stratégie va rompre avec la politique touristique jusqu'alors poursuivie et va faire entrer le pays dans une nouvelle ère de stratégie de développement touristique baptisée l'ère des visions. 
Les visions « 2010 » et « 2020 » de développement touristique : une lecture croisée:-

$\mathrm{Au}$ vue de l'importante croissante que connaissait le tourisme mondial et possédant des atouts naturels importants et un patrimoine culturel riche et diversifié et conscient du rôle déterminant du secteur dans le développement économique du pays, le secteur touristique au Maroc va, à partir de l'année 2001, connaitre un grand changement dans sa politique de développement et de promotion à travers le délaissement des politiques promotionnelles ponctuelles en faveur de la mise en place d'une vraie stratégie de développement touristique volontariste susceptible de déclencher une dynamique de développement durable et intégrée. Cette nouvelle ère baptisée l'ère des «Visions» va marquer clairement le point de rupture avec le passé en matière de politique touristique marocaine en érigeant le tourisme en priorité économique nationale et en dotant le secteur d'une stratégie prospective avec une vision à long terme confortée par des contrats programmes chiffrés et détaillés. La première vision dite «Vision 2010», lancée lors des premières assises du tourisme tenues à Marrakech le 10 janvier 2001 et marquée par la signature de l'Accord Cadre entre le Gouvernement et la Confédération Générale des Entreprises Marocaines (CGEM) et par la suite de la signature de son Accord d'Application, contractualisant ainsi l'engagement des deux parties (publique et privée) de mettre en ouvre le dispositif stratégique de la nouvelle politique touristique, a défini des objectifs très ambitieux aussi bien en termes quantitatifs que qualitatifs.

Tableau $\mathbf{n}^{\circ} 1$ :- Les objectifs de la vision 2010

- Entrées aux frontières : 10 millions

- Capacité hôtelière additionnelle : 160000 lits

- $\quad$ Nuitées touristiques dans les hôtels classés : 50 millions

- $\quad$ Taux d'occupation : $70 \%$

- $\quad$ Emploi additionnel : 600000 emplois

- Contribution du tourisme au PIB: elle devrait progresser en moyenne annuelle de $8.5 \%$, ce qui la porterait à près de $20 \%$ à l'horizon 2010

Source : Vision 2010, Ministère du tourisme marocain.

Cette vision a permis une grande avancée dans le secteur touristique national et a pu insuffler une grande dynamique surtout en matière d'investissement et de réalisation de stations touristiques. Toutefois les objectifs en matière d'arrivées touristiques et de taux d'occupation n'ont pu être pleinement atteints. Cela est dû à la conjugaison de plusieurs facteurs d'ordre conjoncturel et structurel (MEF-DÉPF, 2011, p:12). Sur le plan conjoncturel, le secteur touristique national a subi de plein fouet les effets négatifs de la crise économique qui a affecté les principaux pays émetteurs depuis 2008. Sur le plan structurel, malgré les réalisations physiques en matière d'investissement touristique, l'offre touristique marocaine reste peu diversifiée et spatialement concentrée dans quatre grandes villes (Marrakech, Agadir, Casablanca et Tanger) qui concentrent à elles seules plus de 65\% de la capacité totale d'hébergement. De plus, l'offre balnéaire demeure prépondérante dans la palette touristique globale avec une part de $23 \%$, ce qui place le Maroc en concurrence directe avec les pays compétitifs du pourtour méditerranéen.

A la vue des résultats optimums de la Vision 2010, le pays a décidé de reconduire sa stratégie touristique à travers la «Vision 2020 », qui mise sur un développement touristique plus équilibré et diversifié, capable de renforcer l'offre de chaque région et qui serve de mécanisme de croissance pour ces dernières. En effet, en construisant sur les acquis et les enseignements de la Vision 2010, ainsi que sur les perspectives d'évolution du marché touristique mondial de la prochaine décennie, le but central de la Vision est d'assurer le rôle du tourisme comme moteur et levier principal du développement du pays. Pour cela la vision a défini comme ambitions de hisser la destination Maroc en 2020 parmi les 20 premières destinations touristiques mondiales tout en la positionnant comme une destination de référence en matière de développement durable sur le pourtour méditerranéen.

Tableau $\mathbf{n}^{\circ}$ 2:- Les objectifs de la vision 2020

- Doubler les capacités d'hébergement, avec la construction de 200.000 nouveaux lits.

- Atteindre la barre des 20 millions de touristes issus de différents marchés émergents

- accroîtrons les recettes touristiques pour atteindre environ 140 milliards de dirhams en 2020

- accroîtrons de deux points la part du PIB touristique dans le PIB national,

- Porter la part du tourisme interne à $40 \%$ des nuitées.

- Créer 470000 nouveaux emplois

- $\quad$ et monter à la 20ème destination touristique mondiale.

Source: Vision stratégique de développement touristique «VISION2020», Contrat-programme 2011-2020, 30 Novembre 2010. 
Comme feuille de route pour la réalisation de cette ambition et de ce positionnement, la vision cherche à offrir aux touristes des expériences variées, authentiques, d'un niveau de qualité répondant aux meilleurs standards internationaux, qui valorisent les ressources naturelles et socioculturelles du pays à leur juste potentiel, qui permettent aux populations locales de participer pleinement à l'activité touristique et qui soient respectueuses de leur environnement et de leur qualité de vie. Pour ce faire, aux deux principaux pôles d'activité que sont Marrakech et Agadir, sont venus s'adjoindre plusieurs nouveaux pôles touristiques, dont la montée en charge devrait permettre d'intéresser de nouveaux segments de touristes, de rééquilibrer l'activité touristique et d'en diffuser les bénéfices économiques sur l'ensemble du territoire. La Vision 2020 s'est appuyée ainsi sur une démarche volontariste et intégrée d'aménagement territorial visant d'une part à permettre la mise en valeur des potentialités de chaque région et d'autre part à exploiter toutes les synergies possibles entre les régions, afin que dans leur ensemble, les régions du pays soient plus compétitives et plus visibles sur les marchés internationaux. Cette démarche devrait être rendu possible grâce au concept de territoire touristique (la vision à retenu huit territoires touristiques) défini comme une zone géographique présentant une offre touristique complète, une forte cohérence et identité propres qui en font son originalité, et une masse critique en termes de capacités d'hébergement, de ressources touristiques et de desserte internationale (CPN- Vision 2020, P :11). Pour opérationnaliser la mise en œuvre de cette stratégie, la vision a prévue de manière détaillée et chiffrée un plan de mise en marche de six programmes d'actions structurants pour un portefeuille diversifié de produits adossé à un dispositif intégré de développement d'un tourisme durable, une promotion et une desserte aérienne en adéquation avec l'ambition des territoires, des mesures de soutien pour une dynamique d'investissement soutenue et durable, un plan de valorisation du capital humain et de formation d'excellence, une stratégie pour la compétitivité du secteur et la professionnalisation des acteurs de la chaîne de valeur touristique et un système de gouvernance claire et adéquat.

La dynamique créée par ces deux visions a pu rapidement donnée ces fruits en permettant des performances remarquables. En effet, les données actuelles montrent une nette progression et amélioration de tous les indicateurs du secteur en perspective de la réalisation des objectifs retenus malgré un environnement géopolitique mondial et régional marqué par les risques et l'incertitude. Ainsi, grâce aux efforts déployés dans les deux visions, le Maroc est classé comme la 26ème meilleure destination mondiale et l'objectif retenu pour 2010 a été atteint à hauteur de $93 \%$ (9.3 millions en 2010de touristes internationaux contre 4.4 millions en 2001) avec une croissance annuelle moyenne de $8.7 \%$, très supérieure à la moyenne mondiale. Cette croissance a continué pour atteindre 10,282 944 millions de touristes internationaux à fin 2015 avec une progression de $+2,4 \%$ par rapport à 2014. Le secteur a aussi été d'une contribution décisive aux grands équilibres macro-économiques. En effet, entre 2001 et 2010, les recettes touristiques (hors transfert des Marocains Résidents à l'Etranger) sont passées de 29 à plus de 56 milliards de dirhams, soit un taux de croissance de $7.5 \%$ par an. Les revenus issus du tourisme sont ainsi devenus la première source de devises du pays, devant les transferts de MRE, et loin devant les autres secteurs de l'économie (phosphate, textile, ...). En 2015, ces recettes en devises générées par les non-résidents ayant séjourné au Maroc (hors transport international) se sont élevées à près de 57,2 milliards de dirhams représentant près de $29 \%$ des exportations des biens et services et le solde de la balance des voyages a couvert $24 \%$ du déficit de la balance commerciale en 2015 . Le secteur représente aussi actuellement le deuxième contributeur au PIB national. En effet, le Produit Intérieur Brut du secteur du tourisme a connu entre 2001 et 2010 une croissance annuelle moyenne de $7.6 \%$, supérieure à celle de l'économie dans son ensemble et a pu gagner 2 précieux points dans le PIB national $(8 \%$ contre $6 \%$ au début de la décennie). En 2015, cette croissance s'est encore confirmée et la part du secteur est passée à $12 \%$ du PIB confirmant ainsi l'importance du secteur. Cette dynamique qu'a et que connait le secteur a eu un impact socioéconomique important, puisque le secteur représente désormais près de 450.000 emplois directs, avec une hausse de $40 \%$ du nombre d'emplois durant la décennie. Tendance qui s'est confirmée à fin 2015 puisque le nombre d'emplois a dépassé la barre de 500000 emplois directs soit prés de 5\% de l'emploi dans l'ensemble de l'économie faisant du secteur le deuxième contributeur d'emplois dans le royaume.

Dans le registre de l'hébergement et suite aux efforts entrepris et aux réalisations des deux visions 2010 et 2020, la capacité hôtelière va connaitre une nette évolution depuis l'année 2000. Ainsi d'une capacité de près de 93000 lits à la fin des années 90, la capacité litière classée a atteint plus de 230628 lits à fin 2015. 
Tableau nº 3:- Chiffres clés du secteur touristique marocain (2015).

\begin{tabular}{|c|c|c|c|c|c|}
\hline $\begin{array}{lll}\text { Part } & \text { dans } & \text { le } \\
\text { PIB } & & \\
\end{array}$ & $\begin{array}{l}\text { Nb. } \\
\text { d'emplois }\end{array}$ & VA & $\begin{array}{ll}\text { Taux } & \text { de } \\
\text { croissance } & \end{array}$ & $\begin{array}{l}\text { Capacité } \\
\text { d'hébergement }\end{array}$ & $\begin{array}{l}\text { Croissance } \\
\text { des recettes } \\
\text { de voyage }\end{array}$ \\
\hline $12 \%$ & \begin{tabular}{lrr}
1200000 & \multicolumn{2}{r}{500000} \\
emplois directs & soit \\
prés de $5 \%$ & de \\
l'emploi & & dans \\
l'ensemble & & de \\
l'économie. & &
\end{tabular} & $8 \%$ & $4 \%$ & 230628 lits & $5,2 \%$ \\
\hline
\end{tabular}

Source : Ministère du tourisme marocain, 2015.

Somme toute, le tourisme occupe une place de choix dans la structure économique et financière du pays et représente un levier considérable pour l'accélération de la croissance socio-économique. Véritable moteur de croissance, le tourisme impacte pratiquement tous les domaines de l'activité économique du Maroc et exerce ainsi une grande influence sur les autres secteurs de l'économie. Toutefois, malgré ces réalisations, la grille de lecture du secteur touristique marocain manifeste encore quelques retards par rapport aux objectifs et ambitions annoncés et notamment un flagrant manque dans la capacité d'hébergement comparativement aux principaux pays et destinations concurrentes.

\section{Cadre théorique et conceptuel:-}

\section{Du management à la Gouvernance des destinations touristiques:-}

Comme le rappelle Lozato-Giotard (2003), la filière tourisme est constituée de la production de divers secteurs d'activité constituant une chaîne de fonctions, base de la chaîne de valeur associée au produit touristique. Une destination touristique ne constitue pas, ainsi, une organisation intégrée, mais le regroupement d'un ensemble de professionnels, simultanément autonomes et indépendants. Le management étant défini comme: «la manière de conduire, diriger, structurer et développer une organisation... l'animation d'un groupe d'hommes et de femmes qui doivent travailler ensemble dans le but d'une action collective finalisée » (Thietard et al. 1999), celui de la destination touristique relève des mêmes considérations et suggère ainsi l'analogie entre une destination et une entreprise. Toutefois, compte tenu des caractéristiques des destinations touristiques, ce management présente certaines particularités. La première particularité du MDT provient même du fait de l'existence d'un grand nombre d'acteurs autonomes, prives et publics aux poids, objectifs et intérêts divergents. La deuxième principale particularité réfère à la nécessité de prendre en compte de nombreux autres acteurs animant le territoire de la destination et qui agissent de manière intentionnelle ou non sur de multiples composantes constituant des ressources pour le tourisme et l'attractivité de la destination et donc une grande part du service touristique global lui-même, ou du "bien support" du service touristique, au sens de la théorie des services (Eiglier et Langeard, 1987). Ces acteurs doivent ainsi être sensibilisés et mobilisés pour la réussite de ce management. Ainsi, le MDT exige la prise en compte de la façon la plus opérante de nombreux acteurs fonctionnellement interdépendant mais souvent indépendants du point de vue organisationnel, avec des logiques propres et d'autres parties prenantes souvent non intéressés par l'activité touristique et ainsi dépasser la simple gestion d'une activité économique dans ses fonctions les plus génériques pour embrasser d'autres fonctions liées à la mobilisation, la coordination et l'implication. De ce fait, compte tenu de la grande multiplicité et l'importance des différentes parties prenantes présente sur le territoire de la destination et influençant son développement, le management de la destination n'impliquant que les acteurs touristiques, doit intégrer la dimension coordination entre tous les acteurs et ainsi ce management doit s'étendre pour s'insérer dans un contexte de gouvernance de la destination; celle-ci impliquant tous les acteurs au sein de la destination (Zhang et Zhu, 2014).

\section{De la gouvernance des destinations touristiques:-}

Concept ancien (Stoker, 1998), la notion de gouvernance, issue de l'économie institutionnelle, dans les années 1930 où elle désignait le comportement de l'organisation face à un environnement complexe, elle est devenue, depuis les années 1980, une expression qui s'insère dans les sociétés et les économies d'une façon controversée: à la fois une notion instable et en évolution, elle est aussi une pratique managériale (Paquet, 2008), un outil mobilisateur des acteurs (Letourneau, 2009) pour les territoires et les organisations confrontés aujourd'hui à une féroce compétition internationale. Repris d'abord par les politologues le concept de gouvernance exprime l'idée d'une transformation de l'action publique faisant intervenir de nouveaux acteurs et recourant à de nouvelles modalités de coordination (Borraz et Le Galès, 2001), il va connaitre une grande évolution en termes des disciplines qui l'étudiaient, telles que 
les sciences politiques, le management des organisations (Ruhanen et al.,2010) et la géographie (Leloup et al., 2005) et un foisonnement d'études et de recherches vont lui être consacrées pour en cerner le sens, les caractéristiques et les dimensions. Parmi les nombreuses définitions données à ce concepts celles les plus citées nous retenons la définition fournie par P. Le Gales (1995) qui considère la gouvernance comme «l'ensemble des arrangements et relations, formelles et informelles entre intérêts publics et intérêts privés, à partir desquels sont prises et mises en œuvre des décisions. » et celle de Benko (1992) pour qui «la gouvernance renvoie à des formes intermédiaires de régulation, ni marchandes, ni étatiques, qui articulent intérêts privés et publics, aspects sociaux et économiques. ». En sciences de gestion, la gouvernance a été définie comme «l'ensemble des mécanismes qui ont pour effet de délimiter les pouvoirs et d'influencer les décisions des dirigeants, autrement dit, qui 'gouvernent' leur conduite et définissent leur espace discrétionnaire» (Charreaux, 1997 in Charreaux et Wirtz, 2006,), ce que Pérez (2003) résume par «le management du management». Les systèmes de gouvernance sont alors supposés influencer le processus de création de valeur par le biais, notamment des décisions d'investissement et de répartition de la richesse créée. Dans ce domaine, le cadre théorique dominant la recherche en gouvernance est fortement inspiré des théories contractuelles de la firme, en particulier la théorie de l'agence, la théorie des coûts de transaction et théorie des parties prenantes.

Le tourisme approché comme une filière liant une grande multitude d'acteurs indépendants mais aussi très interdépendants constitue un contexte intéressant dans 1'étude de la gouvernance de la destination (Ruhanen et al. 2010). Toutefois, malgré, la multiplication d'approches n'a pas pu jusqu'alors aboutir à une définition universelle de la gouvernance des destinations et à un corpus théorique propre bien établi. Cela est dû en grande partie à la diversité des champs de recherche des chercheurs et du caractère des études consistant en grande partie en des études de cas qui ne tiennent compte que des dimensions appropriées au contexte de l'étude en question (Beritelli et al. (2007). De nombreux autres auteurs se sont intéressés à l'étude de la gouvernance des destinations touristiques (Beritelli et al, 2007, Ruhanen et al, 2010, Laws et al. 2011, Zhang et Zhu, 2014). En se basant sur les meilleures micro-théories de la gouvernance (théorie des coûts de transaction, théorie des droits de propriété, théorie d'agence, théorie des réseaux), Beritelli et al. (2007) ont pu identifier six dimensions principales de la gouvernance de la destination: «coût de transaction», «asymétrie du pouvoir», «interdépendance», «confiancet/contrôle», «connaissance» et «relations personnelles informelles». Pour mieux opérationnaliser leur analyse, ces auteurs ont retenu d'autres dimensions non moins importantes, notamment : l'histoire de la destination et son développement, la taille de la destination, les performances de la destination, l'existence d'un groupe privé dominant, le rôle de la commune et de l'organisme du management de la destination(DMO), les facteurs d'attractivité, le comportement de coopération, la confiance mutuelle et le climat interne.

De leur coté et sur la base d'une recherche menée sur 53 articles traitant de la gouvernance dans deux discipline majeurs à savoir les sciences politiques et les sciences de management Ruhanen et al, (2010) ont cherché à relever les dimensions de la gouvernance de la destination les plus cités et utilisées. Ainsi, sur une base de 40 dimensions soulevées et en ciblant celles qui étaient citées au moins une dizaine de fois dans ces articles, ces auteurs ont pu retenir les principales dimensions suivantes : imputabilité, transparence, implication, structure, efficacité et pouvoir. En ce contentant uniquement d'énumérer ces dimensions sans pour autant montrer leur importance eu égard à la gouvernance de la destination, ces auteurs concluent que malgré l'existence d'un cadre théorique relativement construit, le concept de la gouvernance de destination reste multidimensionnel. Cette multi-dimensionnalité nous pousse à considérer la destination comme un système constitué par plusieurs acteurs et dans lequel chaque acteur exerce une certaine influence et est influencé par ledit système. D'où la nécessité de faire appelle à l'approche systémique pour analyser la gouvernance de la destination (Laws et al. 2011:10). Cette conclusion est confortée par celle de Zhang et Zhu (2014:125-126) qui affirment que la gouvernance de la destination se caractérise par la complexité due à l'interaction entre les secteurs public et privé, lesquels sont interdépendants en termes de ressources. Pour ces auteurs, complexité, interdépendance sur les ressources et relations entre le public et le privé sont les trois principaux aspects qui caractérisent la gouvernance de la destination.

Somme toute, les différents travaux ayant touché à la gouvernance des destinations attestent de sa grande complexité et de la difficulté de saisir toutes ses dimensions voir encore de juger de son efficacité. Sur cette base plusieurs modèles du management et de la gouvernance des destinations touristiques ont été développés et discutés sur la base d'expériences en vogue dans les pays du nord notamment aux Etats Unis et en Europe. Dans le point suivant nous exposerons les modèles les plus importants et nous discuterons de leur pertinence et efficacité. 


\section{Les modèles de Management et de la gouvernance des destinations touristiques:-}

Avec l'importance de plus en plus grandissante du tourisme dans les économies et dans les stratégies de développement des pays ces trois dernières décennies, la question du management et de la gouvernance des destinations touristiques a retenue l'attention tant des chercheurs, des professionnels, des gouvernementaux et des organismes internationaux (OMT, OCDE). Dans ce registre plusieurs modèles de Management de destination touristique ont été proposés. L'un de ces modèles qui reste le plus répandu et le plus cité est celui développé par les deux fameux auteurs américains Crouch et Richie (Crouch et Ritchie, 1999; Ritchie et Crouch, 2000). En prenant comme référence les travaux développés par $\mathrm{M}$. Porter sur la compétitivité, ces deux auteurs ont élaboré un modèle présentant les éléments constituant la destination touristique et les principales fonctions que le ou les organes de gestion de la destination (DMO) doivent accomplir pour lui assurer réussite et compétitivité. Pour ces auteurs, les organes responsables de la gestion de la destination touristique ont à leur charge deux types de fonctions : celles dites du «management simple » (gestion des ressources, marketing, organisation...) et celles dites « politiques » ou de «management stratégique » (délimitation du système, élaboration d'une vision partagée, définition d'un positionnement, définition des choix stratégiques de développement et des choix de coopération-compétition, définition procédés de diagnostic, et de contrôle-évaluation). En empruntant la voie à ces deux auteurs, Flagestad et Hope (2001) vont développer un autre modèle aussi important et aussi répandu que celui de Crouch et Richie. Considérant la nature et les spécificités des destinations touristiques caractérisées par leur complexité et l'existence de nombreux intervenants et acteurs à intérêts divergents référant à un jeu entre des forces ayant pour objectifs prioritaire la satisfaction des résidents (collectivités publiques) et celles d'autres orientées vers la satisfaction d'objectifs économiques(les entreprises), ces deux auteurs ont tenté d'articuler, malgré leur rivalité, les grandes approches de la stratégie que sont celle du positionnement, celle des ressources et compétences, et sur d'autres plans, celle de la chaine de valeur de Porter pour mettre en exergue la difficulté d'aboutir à un système de management concerté et coopératif entre les acteurs de la destination. Sur ce, les deux auteurs vont dans le sens de proposer un système de management de la destination hybride (dit aussi d'organisation en réseau) se situant entre celui hiérarchique (dominance d'un acteur fort) et celui du marché. Leur modèle va retenir l'attention de nombreux autres auteurs qui l'ont enrichi et élargi via l'intégration de la théorie des parties prenantes en proposant la prise en compte des autres acteurs opérants dans le territoire de la destination et leur nécessaire mise en réseau dans une vision politique du système (Gundolf, Jaouen et Loup, 2006, Dredge, 2006).

Les deux modèles proposés par Crouch et Richie et Flagestad et Hope ont été à la base d'une florissante littérature sur les modes d'organisation, du management et de gouvernance des destinations touristiques qui va permettre de générer et de développer, sur fond de deux théories qui se présentent rivales dans la littérature mais d'apparence complémentaires dans la réalité : les théories contractuelles et la théorie des parties prenantes, deux principaux corpus théoriques présentant modèles extrêmes du management et de la gouvernance des destinations à savoir le Corporate model et le Community model, entre les quels plusieurs situations peuvent se présenter.

Le corporate model (ou modèle entrepreneurial ou modèle d'entreprise) est celui le plus fréquent dans l'Amérique du Nord et repose sur les principes et les postulats des théories contractuelles. Ce type de modèle épouse le schéma d'une «business corporation » selon Flagestad (2001) et considère la destination touristique comme une entreprise dominée par une grande firme ou un groupe privé qui cherche à atteindre des objectifs d'efficience et de profit. Agissant au non de la communauté locale et détenant le pouvoir politique et de décision sur les modalités de développement et d'exploitation de la destination, la firme dominante applique une gestion de type intégrée pour la destination sous fond d'une logique de profit avec une stratégie orientée marché et l'intégration des entreprises de services soit par incorporation dans le groupe soit par contractualisation. Plusieurs avantages sont associés à ce type de modèle. D'une part, la recherche de la maximisation du profit étant l'objectif principal, malgré la présence d'autres petites et moyennes entreprises agissant indépendamment de la grande entreprise, c'est cette dernière qui détermine l'orientation stratégique ainsi que les objectifs principaux de la destination et son pouvoir et sa capacité de mobiliser les ressources nécessaires et de solidifier le réseau d'entreprises incorporées ou liées permet de minimiser et de maitriser les coûts et de réaliser des performances importantes pour toute la destination et d'assurer ainsi le rôle de leader pour les autres acteurs de la destination. (Flagstad et Hope 2001, p. 451-453) D'autre part, en rappelant l'hypothèse émise par Powell (1990) selon laquelle les entreprises hiérarchiquement organisées sont plus susceptibles d'entreprendre des actions (R\&D, Investissement,...) impliquant une incertitude quant à leur résultat et qui nécessitent un investissement substantiel de ressources (argent, temps et énergie), ce type d'organisation permet le développement de l'apprentissage organisationnel nécessaire au renforcement de la position de l'entité et plus important encore pour le processus d'innovation dans la destination étant donné que les investissements dans les innovations de produits, de marchés et de procédés sont un aspect essentiel pour maintenir la compétitivité d'une 
destination. Un autre avantage certain de ce type de modèle, mentionné par Powell (1990), est celui relatif à la question de l'opportunisme. Cet auteur note, en effet, que l'opportunisme des acteurs est atténué par le pouvoir d'autorité exercé par la grande firme. Il avance aussi que les différentes parties ont tendance à développer une identité plus forte quand elles sont regroupées ou liées au sein d'une même société. Ainsi, pour le cas des destinations touristiques gérées dans le cadre du Corporate Model cela se traduit par une diminution du risque d'être affaibli par les comportements opportunistes et déloyaux des acteurs et prestataires et une plus grande identification des acteurs individuels avec une entreprise fortes renforce l'identité de toute la destination. Et cette forte identité permet à touts les acteurs de développer des comportements cohérents qui auront un impact positif sur l'expérience du visiteur et par conséquent sur la compétitivité de la destination.

Le principal inconvénient associé à ce modèle réside dans le risque que la société dominante elle-même tend à agir de manière opportuniste en tirant parti du déséquilibre de pouvoir existant entre elle-même et les petites entreprises indépendantes et les autres acteurs dans la destination. En effet, la recherche de la maximisation du profit pourrait pousser cette société ou groupe dominant à exploiter son pouvoir et son influence pour ne servir que ses propres intérêts ou ne pas remplir ses obligations envers la communauté.

A l'opposé le modèle dit communautaire (community model) très spécifique à l'Europe repose sur la théorie des parties prenantes. Ce modèle considère que la destination touristique est très fragmentée et est constituée d'un grand nombre d'unités de productions de tailles et d'activités différentes, indépendantes, opérant dans une démarche décentralisée, sans pouvoir de domination mais aussi d'autres parties prenantes dont les acteurs publics, les collectivités territoriales et d'autres groupes d'intérêts distincts opérant dans le territoire. Ce modèle, se caractérise donc par la présence de nombreuses parties prenantes qui doivent pour une meilleure gestion parvenir à de fréquents compromis en raison de leurs divergences d'intérêts. Contrairement au premier modèle, le modèle communautaire ne reconnait pas la dominance d'une unité ou d'un acteur sur les autres et le management stratégique des destinations ayant ces caractéristiques posent la problématique de la gouvernance de ces différentes parties prenantes. En général, au vue de la grande multitude des intervenants, la réglementation de ce modèle s'effectue de manière décentralisée, en vertu de laquelle l'organisation locale de gestion des destinations est responsable de la coordination des membres individuels, du marketing et de la formulation de l'orientation stratégique. Cependant, l'influence de cette organisation sur les mesures réelles dans la destination et son pouvoir décisionnel sont limitées. La caractéristique d'une destination communautaire est le fait que ni une entreprise dominante ni l'organisation de gestion ne contrôlent les unités d'affaires de manière hiérarchique.

Comme le font remarquer Flagestad et Hope (Flagestad et Hope 2001, p. 452) dans un tel système les forces politiques sont plus fortes que la vision économique. Et l'organisation du management de la destination dépend plus du comportement coopératif des entreprises indépendantes et des acteurs de gouvernance locale.

Caractérisant deux systèmes différents, ces deux modèles donnent l'apparence qu'ils sont exclusifs. Certes, chacun des deux modèles apporte des éléments d'analyse importants: le corporate modèle mis plus l'accent sur l'importance de l'existence d'un groupe privé fort tant sur le plan financier que sur le plan de la culture d'entreprise adoptant une logique de gestion d'entreprise comme moyen pour assurer la performance et la compétitivité de la destination alors que le modèle communautaire introduit le rôle fondamental des collectivités locales à coté des autres prestataires de services touristiques et la nécessité d'un bon système de gouvernance pour assurer la compétitivité de la destination. Cependant à la question centrale «quel modèle est le plus pertinent en termes de stratégie et de compétitivité ? », la réponse ne peut en aucun cas être à l'absolu. Et pour cause, en retenant la conclusion fondamentale d'Arvid Flagestad (2001) qui statue sur l'existence d'un continuum de situations entre le corporate model et le community model et qu'il ne s'agit pas de se placer dans l'un ou l'autre de ces deux modèles. Dans les destinations caractérisées par la présence de nombreuses entreprises à finalité économique et commerciale et sans réelle domination et de plusieurs acteurs territoriaux gérant les biens publics et garants de l'intérêt général, aucun des deux modèles ne peut être appliqué totalement. Ainsi, dans ces types de destinations, le système d'organisation et de management est à bâtir dans une logique de coopération et de partenariat entre les unités économiques et le pouvoir politique local. En effet, les premiers étant incapables d'assurer le leadership total dans la destination et les second aussi à eux seuls ne peuvent pas non plus assurer la dynamique économique de la destination, une nécessaire coordination et collaboration doit être développée et mise en œuvre entre ces deux sphères publique et privée pour pouvoir mettre en place un système de management et de gouvernance adapté et efficace. Ainsi, compte tenu de ces considérations, il est peu probable que les systèmes d'organisation et de management des destinations observables dans la réalité soient une forme extrême. De ce fait, entre ces deux 
modèles tout un ensemble de situations hybrides est possible et chaque destination doit définir son modèle en fonction de ses spécificités et de la complexité ou non de son contexte et de sa situation.

Analyse du modèle de gouvernance des destinations touristiques au Maroc:-

L'analyse du système de gouvernance touristique des destinations touristiques au Maroc dans le cadre de la nouvelle stratégie de développement touristique sera menée en deux temps. Dans un premier temps nous tenterons de caractériser le modèle promu à travers sa présentation selon le CPN. Dans un deuxième temps nous procéderons, à une lecture de son opérationnalisation à travers l'analyse de son processus de mise en œuvre et son état actuel à quatre années de l'horizon 2020.

Présentation et caractérisation du nouveau modèle de gouvernance des destinations touristiques au Maroc:Jusqu'à l'avènement de la nouvelle stratégie touristique, que nous avons qualifié de l'ère des visions, le secteur touristique était géré en grande partie par les pouvoir publics dans le cadre de plan de développement dont l'Etat définissait la politique et les plans d'action et en assurait la mise en œuvre, le suivi et la régulation sur le plan national et régional. C'est avec la vison 2010 que les premiers jalons de la gouvernance touristique vont être posés à travers une démarche de conception et de mise en œuvre basée sur la concertation et le partenariat comme cadre et principes fondateurs. Cette volonté d'asseoir une meilleure gouvernance s'est traduite aussi dans le cadre de cette vision par la modernisation de certaines structures publiques et la création d'autres. Ainsi, dans le cadre de cette vision, l'Etat a procéder à la modernisation de l'Office National Marocain du Tourisme (ONMT) par son recentrage sur son métier de promotion nationale et du marketing et en le dotant de moyens conséquents, la création par le Ministère du Tourisme de la Société Marocaine d'Ingénierie Touristique (SMIT), en remplacement de la Direction des Aménagements et des Investissements (DAI) pour soutenir et orienter le développement de l'offre, assurer également le suivi des grands projets de la Vision 2010, la création des Conseils Régionaux du Tourisme (CRT), instances regroupant les professionnels privés, les élus et les autorités locales, pour poser les premières bases d'une gouvernance locale, la création de l'Observatoire du Tourisme, pour professionnaliser la publication de statistiques et d'études et la tenue annuelle des Assises du Tourisme pour effectuer le bilan des réalisations et débattre des priorités à venir. Dans cette foulée, l'Etat a aussi procédé, à partir de l'année 2002, à la création des Centres Régionaux d'Investissement (CRI) dont la mission est principalement de centraliser et de simplifier les démarches administratives permettant la création des entreprises et de soutenir et d'accompagner les investisseurs au niveau régional. Concernant le privé, la FNT, acteur majeur représentant le secteur privé, visait d'une part le regroupement en son sein de l'ensemble des professions touristiques et d'autre part le renforcement des structures des différentes professions pour plus d'efficience et de participation aussi bien au niveau régional que national (FNIH, FNAVM, FNR,STT, ALASCAM et FNAGAM). Malgré cela nous pouvons soulever que la gouvernance est restée le parent pauvre de cette vision. Et pour cause les efforts étaient plus concentrés sur l'accentuation de la réalisation des investissements programmés dans le cadre du Plan Azur (programme de réalisation des stations balnéaires) et sur le développement de l'offre culturelle. Ainsi, les efforts étaient plus centrés sur le développement de l'offre en matière de produits (balnéaire et culturel) et sur ce plan l'Etat a pu en grande parti gagner le pari au vue des réalisations physiques enregistrées et a pu ainsi mobiliser les investissements et les acteurs et créer une dynamique positive dans le secteur. Dans son rapport publié en mai 2008, intitulé « Bilan stratégique de la vision 2010» la FNT, atteste que globalement le chantier "organisation institutionnelle" a accusé beaucoup de retard avec un déficit de gouvernance et faiblesse des organes de pilotage ainsi qu'une faiblesse des capacités de gestion des associations du secteur (CRT, Fédérations, Associations professionnelles,...).

C'est avec la vision 2020 que l'on peut parler d'un véritable système de gouvernance dans le tourisme marocain. En effet, reconnaissant la nature transversale du tourisme et son importance à l'échelle nationale les concepteurs de la vision ont cherché à asseoir un fort leadership et une gouvernance renforcée afin d'une part de renforcer la légitimité institutionnelles du secteur (vue la multiplicité des acteurs) et de faciliter les prises de décision au niveau national et régional à travers un pilotage national stratégique de la vision et la garantie de la bonne exécution des stratégies et plans d'actions territoriaux (CPN, p :30). En soubassement à cette logique, l'objectif est de mettre en place des mécanismes et des moyens de pilotage adaptés, mieux partagés et plus synthétiques pour éviter un nombre d'acteurs trop importants (FNT, 2008, p28). Pour ce faire, de nouvelles structures programmées dans le cadre de cette vision se sont venues s'ajouter aux autres structures nationales, régionales et locales déjà en place. Il s'agit principalement de la Haute Autorité du Tourisme (HAT) (dénommée initialement : Conseil National du Tourisme) et des Agences de Développement Touristique (ADT). D'après la Mesure 37 du CPN (CPN-Vision 2020 ; p : 30), la HAT est une instance nationale de pilotage, réunissant dans un partenariat renouvelé l'Etat, les régions et le secteur privé qui aura pour principales missions de s'assurer de la cohérence de la vision à long terme et du suivi de son exécution, de 
suivre et évaluer l'action des différents acteurs clés publics et privés de la gouvernance du tourisme ainsi que de suivre et évaluer l'exécution des feuilles de routes régionales en veillant en particulier à un pilotage coordonné de tous les paramètres clés de l'équation touristique, à la régulation dynamique des capacités hôtelières et de formation des ressources humaines; d'assurer le suivi des efforts des autorités concernées pour une adéquation entre les capacités aériennes et les capacités litières et de proposer les ajustements nécessaires à la mise en œuvre de la Vision et le cas échéant à la révision de ses paramètres stratégiques. Véritable organe de pilotage et de gouvernance du secteur au niveau national la HAT, dont la mise en place était prévue pour au plus tard à la fin de l'année 2011, est composée, sous la tutelle du Ministère du Tourisme, qui en assure le Secrétariat Général, une dizaine d'autres ministères, la CGEM et 5 représentants du secteur privé (représentants des fédérations professionnelles). Pour accompagner et réussir la régionalisation touristique retenue dans la vision 2020 et de s'assurer de la mise en œuvre des stratégies territoriales (CPR), la HAT sera reliée sur le plan régional par les ADT qui devraient êtres progressivement créées dans les 8 territoires touristiques retenus (4 agences à fin 2011 et 4 agences à fin 2012). Ces agences, d'après la mesure 38 du CPN (CPN-vision 2020; p : 31), devraient avoir pour principales missions de garantir la bonne exécution des Contrats Programmes Régionaux (CPR) de leur territoire respectif pour en favoriser un développement touristique harmonieux, rapide, pérenne et rentable et ce à travers, outre la déclinaison des missions de la HAT au niveau régional, de développer l'attractivité globale des territoires, en direction des investisseurs et grands acteurs du secteur, de développer la compétitivité des territoires via la coordination, d'assurer la promotion, la commercialisation et l'accompagnement du développement de la desserte aérienne de leur territoire respectif auprès des marchés ciblés, d'assurer le contrôle de la qualité sur l'ensemble de la chaîne de valeur et au sein de l'environnement touristique, d'assure le suivi et l'orientation des projets stratégiques et d'orienter les professionnels locaux du secteur en fonction des tendances du marché mondial ainsi qu'une diffusion des meilleures pratiques et des démarches novatrices.

Ainsi, dans cette nouvelle configuration, les ADT assureront le rôle du principal interlocuteur des acteurs publics et privés pour toutes les questions liées au tourisme, et constitueront une plateforme d'échange et de concertation au niveau de leur territoire. Dans ce schéma les structures actuelles, notamment la Délégation Régionale du Tourisme, les Conseils Régionaux du Tourisme (CRT) et les Conseils Provinciaux du Tourisme (CPT) seront remplacés progressivement par les ADT des territoires concernés. En matière de gestion et de fonctionnement, ces ADT, étant des établissements publics sous la tutelle du Ministère du Tourisme, seront pilotés par un Conseil d'administration, composé de représentants des secteurs public et privé (MT, les représentants des Ministères, les élus et les représentants des associations professionnelles régionales relevant du territoire) et opéreront par des «contrats de performance» pluriannuels validés et suivis par la HAT. Dans leurs activités opérationnelles les ADT auront la tache d'une part, en collaboreront avec les centre régionaux d'investissement (CRI), de développer de l'offre produit local en cohérence avec la stratégie nationale, d'orienter et d'accompagner les investisseurs spontanés et de piloter et d'assurer le suivi des projets planifiés. Et d'autre part d'élaborer et de mettre en œuvre une Stratégie Intégrée de promotion et de Marketing Territorial en parfaite cohérence avec la stratégie de marque nationale.

En référence aux modèles théoriques présentés auparavant, et en plaçant la gouvernance basée sur le partenariat public-privé comme pilier fondamental de la mise en œuvre et de la réussite de la nouvelle politique touristique, le système de management et de gouvernance touristique marocain s'apparente plus à un modèle hybride entre le corporate modèle et le community modèle avec un rapprochement de ce dernier. En effet, en misant sur les ADT comme organe de management et de territorialisation de la mise en œuvre de la stratégie de développement touristique le modèle marocain va dans le sens de l'émergence d'un acteurs collectif au sens de Gerbaux (Gerbaux 2003) ou d'acteur stratégique (Gerbaux et Marcelpoil, 2003) composé de différentes parties prenantes, publiques et privées et qui est propres aux destinations organisées de façon communautaires. Toutefois dans un environnement rapidement évolutif caractérisé par le déplacement des barrières entre acteurs publics et privés ce modèle de gouvernance ne pourrait réussir que si trois principaux éléments sont réunis : la capacité d'agir, l'existence d'acteurs et d'intérêts organisés et des relations entre acteurs afin d'agir ensemble (Le Galès, 1995, pp. 81-82). Pour cet auteur, l'existence d'une réelle coalition d'acteurs publics et privés est la condition nécessaire pour définir et mettre en place des politiques efficaces. Cette avancée est toutefois minimisée par Lorrain (2000) pour qui l'existence d'une coalition n'est pas suffisante pour faire émerger et réussir un acteur stratégique, du fait que gouverner un territoire suppose, selon lui, de parvenir à des compromis entre des intérêts potentiellement divergents, sur un horizon temporel lointains dont les résultats sont aléatoires. Pour cet auteur, ni les institutions politiques en raison des contraintes électorales ni les acteurs privés évoluant dans un environnement de plus en plus concurrentiel et mutant ne sont disposés à s'engager sur ces horizons lointains aux résultats incertains. Ces contraintes risquant de compromettre la mission de l'acteur stratégique l'auteur privilégie la thèse de l'acteur réactif à celle de l'acteur 
stratégique. C. Van Der Yeught (2009) de son coté avance que l'émergence d'un acteur stratégique collectif exige la réunion de trois conditions fondamentales relevant de la gouvernance des destinations à savoir l'hybridation des modes de coordination déployés, la relative indépendance politique de l'équipe dirigeante et sa forte légitimité.

L'hybridation des modes de coordination consiste en le déploiement d'un dispositif de gouvernance permettant d'assurer la coordination des nombreuses parties prenantes impliquées dans le projet productif, de doter le groupe d'une intention stratégique et de maintenir le cap fixé à long terme, indépendamment des jeux de pouvoir et des aléas du calendrier électoral. L'indépendance politique de l'équipe dirigeante est la seconde condition favorisant l'émergence d'un acteur stratégique. Cette indépendance est nécessaire pour ne pas compromettre et entraver le déroulement des projets à long terme. Quand à la légitimité de l'équipe dirigeante elle repose soit sur l'existence d'une réglementation claire ou sur une volonté collective des parties prenantes basée sur des valeurs partagées et une confiance mutuelle. Cette analyse montre que l'émergence d'un acteur stratégique collectif au sein d'une DT ne va pas de soi, mais dépend plutôt de la structure de gouvernance déployée. Qu'en est-il dans le cas du modèle marocain? Les ADT prévues dans le cadre du CPN seront des établissements publics sous la tutelle du MT, constituées par la fusion des Délégations Régionales du Tourisme, des CRT et des CPT. Or la délégation est une administration alors que les CRT et les CPT sont des associations professionnelles avec l'autonomie budgétaire. Cette nouvelle structure abritera en son sein ainsi des fonctionnaires et des professionnels et sera régie par les règles du droit administratif et des finances publiques. Au regard des trois exigences d'une gouvernance efficace énoncées ci-dessus il parait très difficile que les ADT deviennent l'acteur stratégique collectif au sens de Van Der Yeught. Une faible légitimité étant donné la faible représentation de la sphère privée pierre angulaire de la mise en œuvre de la vision, une faible indépendance de l'équipe dirigeante à cause de prédominance de la sphère publique et politique dans la gestion et le financement et un problème de gestion étant donné les divergences des visions et des compétences en matière de gestion et de prise de décision entre les fonctionnaires et les gestionnaires issus des structures fusionnées et par conséquent un risque majeur d'émergence de problèmes de coordination et d'adhésion de tous les acteurs aux intérêts divergents.

Après avoir présenté et caractérisé le modèle de gouvernance des destinations touristiques marocaines dans le cadre de la nouvelle stratégie de développement nous allons dans le point suivant comment cette nouvelle gouvernance s'est traduite dans la pratique après pratiquement 6 années de son adoption et à 4 années de l'horizon des objectifs tracés.

\section{La gouvernance des destinations touristiques marocaines à l'épreuve de la mise en euvre et de l'opérationnalisation:-}

Notre étude de la gouvernance des destinations touristiques marocaines dans le nouveau modèle de développement touristique a été opérée en en deux principaux temps. L'analyse de la gouvernance dans le processus de mise en œuvre de la nouvelle stratégie touristique à travers une lecture documentaire et son opérationnalisation effective sur la base des résultats de notre étude terrain.

En retenant qu'un processus de gouvernance est un «ensemble défini d'activités qui sont effectuées dans le cadre de la gouvernance pour permettre la définition cohérente, l'application et, au besoin, la modification des règles qui organisent et régulent les activités des participants pour mettre en œuvre les politiques énoncées» nous chercherons à caractériser la gouvernance dans le processus de mise en œuvre de la gouvernance dans la nouvelle politique touristique marocaine. Ce processus dans le cas du Maroc à connu trois principales phases. La première phase caractérisée par une implication et participation limitée des acteurs dans le cadre de l'élaboration du CPN et une deuxième phase marquée par une forte implication et participation des acteurs selon une approche up-top dans le cadre de l'élaboration des CPR. La troisième étape, compte tenu du retard accusé dans la mise en place des structures prévues (ADT), est caractérisée par un scénario secours donnant le pilotage régional des contrats.

D'après les données disponibles, c'est en 2009, au vue du bilan provisoire de la vision 2010, que les acteurs concernés (MT et FNT) avec l'aide de bureaux d'études étrangers ont entamé les réflexions et les travaux pour l'élaboration des orientations stratégiques qui devraient aboutir à la vision 2020. Ces travaux qui vont durer près de trois ans n'ont pas été marqués par une implication et participation de tous les acteurs du secteur. L'urgence de présenter la nouvelle stratégie dans les assises du tourisme de 2010 a fait que l'implication et la concertation ce sont plus limitées à l'information des acteurs. Pour nombre de professionnels cette approche n'a pas respecté les principes d'une bonne gouvernance et s'apparente plus à une approche top-down masquée par une tentative d'implication ad hoc des acteurs. 
La vraie démarche de concertation et d'implication de tous les acteurs et donc de gouvernance n'a pu se concrétiser que dans le cadre de l'élaboration des CPR. En effet, si l'élaboration du CPN n'a pas connu, à l'avis de tous et plus particulièrement des professionnels, une réelle concertation et participation, c'est l'élaboration des CPR qui a connu la collaboration et la coordination des différents acteurs impliqués sur les plans local, régional et national selon une approche bottom-up. En effet, le CPN stipule (Mesure 1, CPN p :13), comme l'une de ses recommandations principales pour la mise en œuvre de la vision 2020, que chacun des 8 territoires (ou destinations touristiques) identifiés par la Vision 2020 élabore son Contrat Programme avec des objectifs fixés à partir des potentialités naturelles de chaque région, des pistes de financement pour la construction d'infrastructures et d'une offre touristique spécifique pour accueillir les touristes dont le nombre potentiel est aussi déterminé pour chaque destination. Ces CPR constituent, ainsi, des feuilles de routes précises qui doivent s'élaborer en étroite concertation avec l'ensemble des acteurs locaux: élus, professionnels, société civile et acteurs publics de chaque territoire. La démarche générale d'élaboration des CPR est présentée dans le schéma suivant :

Figure n ${ }^{\circ}$ :- Démarche d'élaboration des CPR

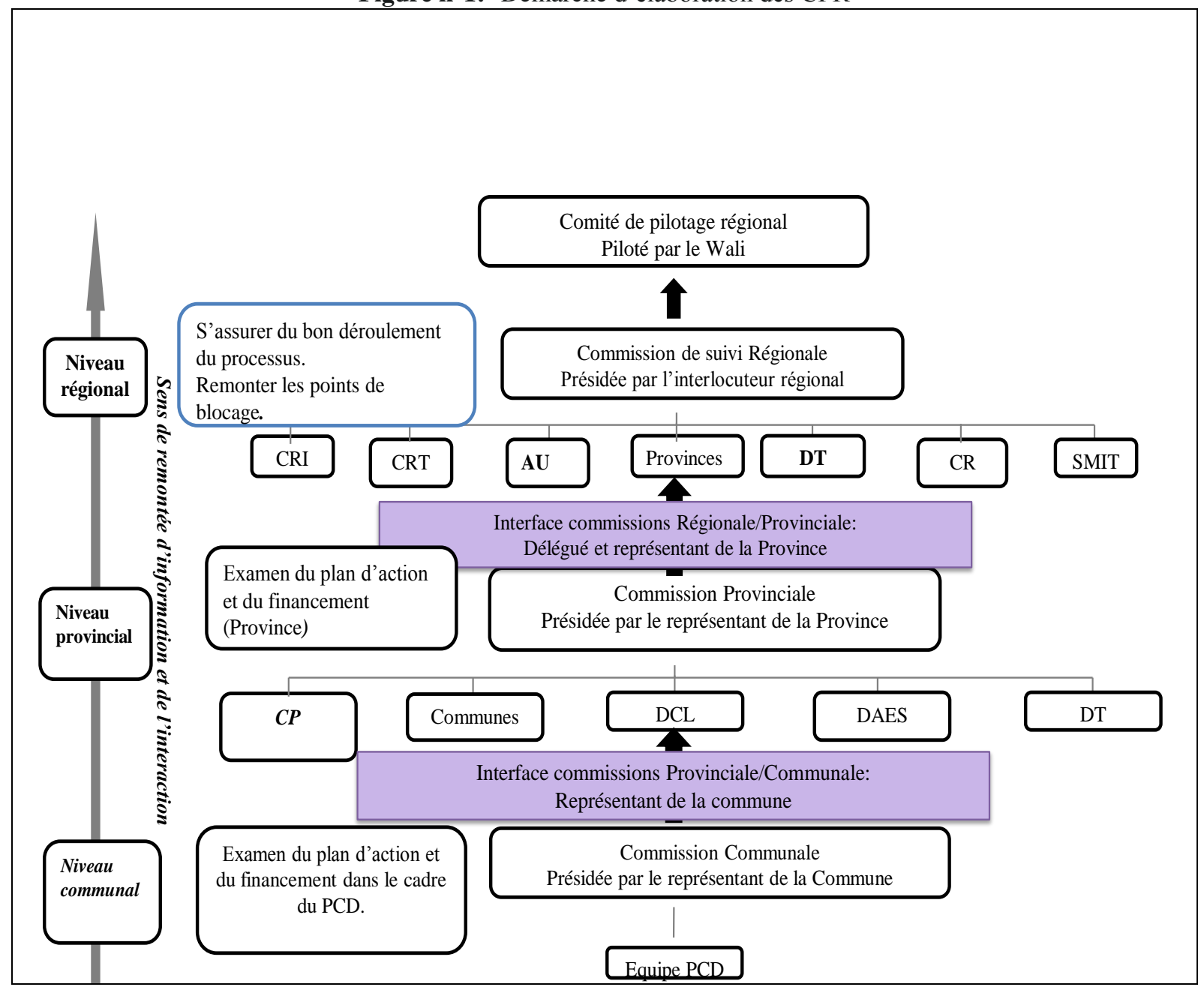

Source : élaboré par les auteurs sur la base du processus décrit dans le CPN.

Suivant une démarche interactive l'élaboration des CPR a débuté par la consultation du niveau local (communes) à travers le listage des projets touristiques retenus dans les plans communaux du développement (PCD) puis leur filtrage au niveau de chaque province en présence du représentant du Ministère (Délégué) et leur validation au niveau régional pour leur mise en œuvre sous forme du programme. le programme validé sur le plan régional est enfin remonté au niveau national pour un dernier filtrage visant à vérifier la concordance et la convergence des projets avec le positionnement du territoire d'implantation tel que prévu dans la vision 2020. 
Si la démarche voulue a été globalement respectée plusieurs limites sont à soulever et qui entravent le processus de gouvernance mais aussi la réalisation des objectifs de la vision 2020. En premier, il ya lieu de noter la grande problématique de non concordance entre les découpages administratif et touristique et leurs mutations dans la période d'élaboration et de mise en œuvre des CPR. En effet, en cherchant à développer une expérience touristique beaucoup plus diverse, tant en termes de nombre de destinations que de variété de l'offre au sein de chacune de celles-ci, la vision 2020 s'est construite sur un découpage, qui transcende les barrières administratives à fin de permettre à chaque région de tirer profit de l'ensemble de ses potentialités et ainsi huit territoires touristiques en été retenus. Toutefois, le découpage administratif de l'époque était composé de 16 régions. Ainsi, 16 CPR étaient prévus pour huit territoires touristiques. D'autant plus que depuis 2011 l'organisation administrative du pays a connu un changement notable en limitant le nombre des régions administratives à seulement 12 régions (régionalisation avancée dont le projet de décret a été adopté par le conseil de gouvernement en février 2015). Dans ce contexte de mutation et du retard de mise en œuvre de la régionalisation avancée et des structures de gouvernance touristique notamment les ADT, chargées de la mise en œuvre territoriale de la vision, confrontés à l'horizon de réalisation des objectifs de la vision, l'élaboration des CPR a été l'œuvre de sous-commissions créées aux différents échelles régionales. Ainsi, encore une fois c'est le découpage administratif et non touristique qui a prévalu étant donné que pour outrepasser les problèmes et les tensions entre territoires et entre acteurs en jeu, c'est à l'échelle des provinces que les projets ont été filtrés pour leur simple validation au niveau de la région. Dans ce processus qui a pris au moins trois années pour l'élaboration des CPR pour toutes les régions nous pouvons ainsi relever la prédominance du politique sur les autres acteurs étant donné que le comité régional est présidé par le wali de la région. Cette situation montre que la régionalisation touristique n'a pas été respectée, ni dans sa dimension territoriale ni dans sa dimension gouvernance, et les CPR, s'apparentent plus à une collection de contratsprogrammes provinciaux loin de la logique d'une réelle régionalisation touristique supposant une vraie appropriation du territoire par tous les acteurs comme espace permettant la résolution de leurs problèmes identifiés comme communs. En effet, les retards accusés dans l'opérationnalisation de la régionalisation avancée et de la régionalisation des structures de gouvernance touristique ont fait que les différents acteurs ont adopté la voie de la collaboration et de la coordination en se référant au territoire administratif et non au territoire touristique.

En second, un autre aspect important de la défaillance de la gouvernance touristique lors du processus d'élaboration des CPR réside dans la manière avec laquelle les projets ont été proposés et adoptés. En effet, alors que la démarche prévoyait donner la priorité aux acteurs locaux pour la proposition des projets pour faciliter leur territorialisation et leur appropriation il n'en été rien dans la réalité, lors des concertations, les représentants régionaux du ministère du tourisme se sont déplacés aux communes avec une banque de projets conçus à l'avance et concordants avec la vision 2020 pour chaque territoire. Cette procédure à faciliter l'alignement de ces communes qui se sont appropriées ces projets et les ont intégré dans leur propres programmes communaux de développement au lieu de l'inverse. Ainsi, la remontée de l'information, le filtrage et la validation des projets et du CPR se sont trouvés faciliter sans réelle concertation et implication de tous les acteurs territoriaux et sans réelle vraie territorialisation des projets.

En troisième, la question de la gouvernance dans le contexte actuel est vécue différemment par les deux sphères publique et privée et les départage. Du coté de la sphère publique la course vers la réalisation des objectifs de la vision 2020 est vécue comme une contrainte majeure et conduit à une sorte de gouvernance de la crise. En effet, après plus de trois ans de l'adoption des CPR et le retard très important accusé dans la concrétisation de la grande majorité des projets d'investissement retenus, une simple analyse de l'agenda des comités du pilotage régionaux, montre la grande priorité consacré au suivi de l'état d'avancement des projets et à la recherche de l'élimination des facteurs de blocage à leur réalisation et à la recherche d'investisseurs preneurs et de financement et les questions du management et de la gouvernance des destinations dans leur aspect promotionnel et marketing sont reléguées au second ordre. Du coté de la sphère privée, la situation est différente. En effet, les professionnels du secteur, acteur majeur dans la mise en œuvre de la vision et des CPR, touchés pour leur grande majorité par la conjoncture difficile que travers le tourisme national, sont plus préoccupés par la situation économique de leurs unités en grande majorité en difficulté. Malgré, qu'ils avancent adhérer sans faille aux politiques et décisions prises (une sorte d'adhésion de façade ou sous silence), ils sont plus demandeurs de mesures de soutien et d'actions orientées vers la promotion de la destination et de la recherche de marchés et marginalisent les préoccupations majeurs des acteurs publics plus orientés vers l'accélération de la concrétisation des programmes retenus dans les CPR. Au sein même de cette sphère privée les avis sont très disparates. Dans presque toutes les destinations du pays compte tenu de la conjoncture difficile que travers le tourisme, nous assistons à une structure à double palier. Alors que les grandes structures hôtelières et plus particulièrement celles appartenant à des chaines internationales se comportent bien la grande majorité des structures indépendantes sont en difficultés et sont plus préoccupées par la recherche de 
solutions commerciales ou économiques à leur problèmes. Dans ce contexte, pour ces acteurs, parler de gouvernance, de collaboration et d'adhésion relève presque de l'impossible.

Somme toute, à quatre années de l'horizon 2020, en mettant de coté les trois seuls points vraiment lumineux dans cette nouvelle politique consistants en le partage de l'ambition par tous et la validité du modèle de gouvernance promu et une forte tendance tant de la sphère publique que celle privée vers une vraie régionalisation de la gestion et de la gouvernance des programmes et des projets touristiques, nous sommes en droit, au vue des grands retards accusés tant dans la mise en place de la régionalisation avancée, des structures de gouvernance touristiques régionales et des projets et programmes retenus, de se poser des questions sur la validité des objectifs retenus et aussi de se poser la question légitime de savoir s'il s'agissait d'une véritable stratégie pour laquelle on s'est données réellement et concrètement les moyens de sa mise en œuvre et réussite ou simplement d'une période transitoire à l'apprentissage du management et de la gouvernance du tourisme dont il faudra tirer les enseignements nécessaires et enclencher les mesures et actions efficaces pour bâtir le vrai cadre d'un management stratégique et de gouvernance efficace de nos destinations touristiques.

\section{Conclusions:-}

En posant la problématique de la gouvernance des destinations touristiques marocaine nous avons cherché à placer le modèle de gouvernance promu dans le cadre de la nouvelle stratégie de développement touristique du pays dans le référentiel théorique mais aussi à le questionner dans le processus de sa mise en œuvre et opérationnalisation. En plaçant la gouvernance basée sur le partenariat public-privé comme pilier fondamental de la mise en œuvre et de la réussite de la nouvelle politique touristique, le système de management touristique marocain s'apparente plus à un modèle hybride entre le corporate modèle et le community modèle. Toutefois, si le schéma institutionnel de la gouvernance tel que décrit dans le CPN semble respecter les principes d'une bonne gouvernance des destinations touristiques, sa traduction dans le terrain a connu des limites. Les réformes territoriales et organisationnelles n'étant pas encore achevées conjuguées à la conjoncture difficile que traverse le tourisme national ne doivent pas pour autant entrainer les acteurs dans un scénario de gestion de la crise mais plutôt d'appréhender et de voir en cette situation une occasion d'apprentissage pouvant engendrer une dynamique positive susceptible d'entrainer des améliorations dans leur manière de percevoir les logiques du secteur et son organisation pour un meilleur management et gouvernance. Chaque destination étant spécifique et ayant son contexte territorial et d'acteurs particulier, seule une gouvernance basée sur le partage d'objectifs communs, la coordination et la concertation de tous les acteurs dans un climat de participation, de cohérence, de confiance, d'ouverture, de responsabilité et d'efficacité peut assurer les bases d'une meilleure compétitivité des destinations touristiques marocaines. Le tourisme est et restera encore et pour toujours un pilier de l'économie marocaine, ré-ouvrir, ainsi, le chantier de la gouvernance, le rediscuter et le réussir à l'échelle territoriale pertinente semble être la voie prometteuse pour asseoir un management stratégique performant pour assoir la compétitivité et la durabilité des destinations touristiques marocaines.

\section{Références:-}

1. Beritelli, P., Bieger, T. et Laesser, C., (2007), «Destination governance: Using corporate governance theories as a foundation for effective destination management», Journal of Travel Research, vol. 46, no. 1, pp. 96-107, 2007.

2. Buhalis, D. (2000), «Marketing the competitive destination of the future », Tourism Management, 2 , pp. 97- 116.

3. Crouch G.I. et Ritchie J.R.B, (1999), «Tourism, competitiveness, and social prosperity», Journal of Business Research, 44, 137-152.

4. CPN- Vision stratégique de développement touristique: Contrat Programme 2011-2020, 30 Novembre 2010.

5. Flagestad A. et Hope C.A. (2000), «Strategic success in winter sports destinations: a sustainable value creation perspective», Tourism Management, $\mathrm{N}^{\circ} 22$, June, pp-445- 461.

6. Gerbaux, F., et Marcelpoil, E. (2006). «Gouvernance des stations de montagne en France : les spécificités du partenariat public-privé », Revue de géographie alpine, 94(1), pp. 9-19.

7. Gerbaux, F., Boudières, V., et Marcelpoil, E. (2004), « De l'utilité de la notion de gouvernance pour analyser les modes de management touristique locaux: l’exemple de la station des Arcs », Ingénieries - EAT(37), pp. 7585.

8. Gerbaux F., Moreau P. (1996), «Le management des stations face au marché touristique, vers une gouvernance locale? », in "La gestion des stations", Les cahiers Espaces, n47, juin 1996, pp 40-48. 
9. Kozak, M., Gnoth, J., et Andreu, L., (2008), «Destination Management and Marketing.» Emerald Group Publishing Limited.

10. Langlois, M., (2004). « Le Management Expérientiel Médiatique (MEM) des organisation de services». Glec.

11. Le Galès, P. (1995) : «Du gouvernement des villes à la gouvernance urbaine ». Revue Française de Sciences Politiques, pp. 57-95

12. Lorrain, D. (2000) : «Gouverner les villes, Questions pour agenda de recherche», Pôle Sud, Qui gouverne les villes? $\mathrm{N}^{\circ} 13$, pp- $27-40$

13. Lozato -Giotart ,J.P. ( 2003), «Géographie du Tourisme: De l'espace consommé à l'espace maîtrisé», PEARSON EDUCATION France, Paris, 2003.

14. Marcelpoil, G.E., et François, H. (2012), «De la construction à la gestion des stations : l'émergence de logiques de groupes dans la vallée de la Tarentaise», Revue de géographie alpine/Journal of Alpine Research, 100(3).

15. MARCELPOIL, G.E. (2012), «Gouvernance et management local des destinations touristiques de montagne - frein ou vecteur pour l'innovation touristique? », Colloque «Les voies de l'innovation touristique», Festival International des Métiers de la Montagne (FIMM) 16 novembre 2012, Chambéry (F).

16. Ministère de l'Economie et des Finances, Direction des Études et des Prévisions Financières (DEPS/SAT), « Secteur du tourisme : bilan d'étape et analyse prospective », Avril 2011.

17. Ministère du Tourisme et de l'Artisanat : «Dossier de presse des 10ème assises du tourisme», Royaume du Maroc, Ministère du tourisme, novembre 2010.

18. Peters M. et Strobl, A. «Conclusion In Contemporary Destination Governance: A Case Study Approach». Published online: 09 Apr 2015; 223-232.

19. Ritchie J.R.B. et Crouch G. I., (2003), «The competitive destination: A sustainable tourism perspective». Cambridge », Cabi Publishing.

20. Ritchie J. R. B. et Crouch G. I., (2000), «The competitive destination: a sustainability perspective», Tourism Management, 21: 1, pp. 1-7.

21. Ruhanen, L. Scott, N., Ritchie B., et Tkaczynski, A., «Governance: a review and synthesis of the literature», Tourism Review, vol. 65, issue 4, pp. 4-16, 2010.

22. Van Der Yeught, $\mathbf{C}$., «Favoriser l'émergence d'un acteur stratégique dans les destinations touristiques pour répondre aux défis du développement durable », Management \& Avenir, 2009/6 (n²6) Pages 300 - 317).

23. Zhang, H. et Zhu, M., «Tourism Destination Governance: A Review and Research Agenda», International Journal of e-Education, e-Business, e-Management and e-Learning, Vol. 4, No. 2, April 2014. 